and also may present a diverse metabolism than men. But whilst the reason why AAPs correlates with LUTS in women only remain obscure it is interesting to notice that SSRIs do not. As such, women under SSRIs treatment who present LUTS should be further investigated instead of having their therapy discontinued.

Dr. Ricardo Miyaoka

State University Campinas

Campinas, SP, Brazil

E-mail: rmiyaoka@uol.com.br

\title{
GERIATRIC UROLOGY
}

\section{Prostate cancer in the elderly: Frequency of advanced disease at presentation and disease-specific mortality}

Scosyrev E, Messing EM, Mohile S, Golijanin D, Wu G

Department of Urology, University of Rochester Medical Center, Rochester, New York

Cancer. 2011; 17. doi: 10.1002/cncr.26392. [Epub ahead of print]

Background: The objectives of this study were to determine the frequency of metastatic (M1) prostate cancer (PC) at presentation in different age groups, to examine the association of age with PC-specific mortality, and to calculate the relative contribution of different age groups to the pool of M1 cases and PC deaths.

Methods: Records from 464,918 patients who were diagnosed with PC from 1998 to 2007 were obtained from the Surveillance, Epidemiology, and End Results (SEER) database. The patients were categorized according to age into groups ages $<50$ years, 50 to 54 years, 55 to 59 years, 60 to 64 years, 65 to 69 years, 70 to 74 years, 75 to 79 years, 80 to 84 years, 85 to 89 years, and $\geq 90$ years. The cumulative incidence of death from PC was computed using the Gray method.

Results: The frequency of M1 PC at presentation was 3\% for the group aged $<75$ years, $5 \%$ for the group ages 75 to 79 years, $8 \%$ for the group ages 80 to 84 years, $13 \%$ for the group ages 85 to 89 years, and $17 \%$ for the group aged $\geq 90$ years. The 5 -year cumulative incidence of death from PC was $3 \%$ to $4 \%$ for all patients with PC in any category aged $<75$ years, $7 \%$ for patients ages 75 to 79 years, $13 \%$ for patients ages 80 to 84 years, $20 \%$ for patients ages 85 to 89 years, and $30 \%$ for patients aged $\geq 90$ years. Although patients aged $\geq 75$ years at PC diagnosis represented just over a quarter $(26 \%)$ of all PC cases, they contributed almost half $(48 \%)$ of all M1 cases and more than half (53\%) of all PC deaths.

Conclusions: Compared with younger patients (aged $<75$ years), older patients were more likely to present with very advanced disease, had a greater risk of death from PC despite higher death rates from competing causes, and contributed more than half of all PC deaths. Awareness of this issue may improve future outcomes for elderly patients with PC.

\section{Editorial Comment}

The recognition of the substantial contribution of older age groups to the pool of M1 cases and prostate cancer (PC) deaths is fundamental to advancement of its treatment and handling. In the presented study, although older patients did not lose as many years of life as younger patients because of shorter remaining life expectancy in the elderly, the proportion of remaining life lost in the elderly still was very high. While it is known that the death rates from competing causes are higher in the elderly, the absolute risk of death from PC increased with age. 
In this scenario of cancer time line paralleling the human aging process there are many irrefutable biases acting on the numbers coming from population based studies such as lead time and selection biases, adding to those for treatment or tumor characteristics.

It is obvious that people that score higher ages are selected from population when they fail of perishing from many other conditions. Authors have speculated more aggressive disease (e.g., faster growing tumors) in the elderly and/or less frequent use of PSA testing and further diagnostic evaluation (such as biopsy for an elevated PSA) in older men compared with younger men. Furthermore, among a compilation of possible biases, two mechanisms were well recognized: 1) over diagnosis of nonaggressive PC in younger patients and 2) delay in diagnosis of aggressive disease in the elderly.

Until nowadays, PC natural history is complex and unpredictable in a large number of cases. The aging process will naturally culminate with death, and it is not surprisingly that virtually all cause of death will increase with age considering the cumulative co-morbidities and the innately decreasing functional status.

While it should be viewed with caution, better understandings of the aging process as well as prostate cancer natural history will add to the understanding of the illustrated scenario.

Dr. Leonardo Oliveira Reis Assistant Professor of Urology University of Campinas, Unicamp Campinas, São Paulo, Brazil E-mail:reisleo@unicamp.br

\section{Clinically relevant fatigue in men with hormone-sensitive prostate cancer on long-term androgen deprivation therapy}

Storey DJ, McLaren DB, Atkinson MA, Butcher I, Frew LC, Smyth JF, Sharpe M

Edinburgh Cancer Research UK Centre, University of Edinburgh, Edinburgh

Ann Oncol. 2011; 17. [Epub ahead of print]

Background: The purpose of the study was to determine the prevalence and associations of clinically relevant fatigue (CRF) in men with biochemically controlled prostate cancer on long-term androgen deprivation therapy (ADT).

Patients and Methods: One hundred and ninety-eight men were surveyed and the prevalence of CRF (Brief Fatigue Inventory score $>3$ ) determined. Associations with other measures (Hospital Anxiety and Depression Scale; International Prostate Symptom Score; European Organisation for Research and Treatment of Cancer Quality of Life Questionnaire; Brief Pain Inventory worst pain; clinical and demographic information) were explored in univariate and multivariate analyses.

Results: Eight-one per cent (160 of 198) of questionnaires were analysable. CRF prevalence was $43 \%$ (68 of 160). CRF associations included moderate/severe urinary symptoms, anxiety and medical co-morbidities; the strongest associations were depression [odds ratio (OR) 9.8, 95\% confidence interval (CI) 4.3-22.8] and pain (OR 9.2, 95\% CI 4.0-21.5). After controlling for other factors, the independent associations were depression (OR 4.7, 95\% CI 1.6-14.0) and pain (OR 3.1, 95\% CI 1.0-8.9). There was no association with age, disease burden or treatment duration.

Conclusions: Two-fifths of men with biochemically controlled prostate cancer on long-term ADT report CRF that interferes with function. Management aimed at improving CRF should address depression and pain. 